\title{
Bone HDR brachytherapy in a patient with recurrent Ewing's sarcoma of the acetabulum: Alternative to aggressive surgery
}

Rafael Martínez-Monge ${ }^{1}$ * Agata Pérez-Ochoa ${ }^{1}$, Mikel San Julián ${ }^{2}$, Dámaso Aquerreta ${ }^{3}$, Luis Sierrasesúmaga ${ }^{4}$

${ }^{1}$ Department of Oncology, University of Navarre, Pamplona, Spain

${ }^{2}$ Department of Orthopedic Surgery, University of Navarre, Pamplona, Spain

${ }^{3}$ Department of Radiology, University of Navarre, Pamplona, Spain

${ }^{4}$ Department of Pediatrics, University of Navarre, Pamplona, Spain

\section{ABSTRACT}

A 17-year-old girl diagnosed with a previously irradiated, locally recurrent Ewing's sarcoma involving the puboischiatic rami and the adjacent acetabulum was referred to our institution for consideration of salvage hindquarter resection. A conservative resection with implantation of the acetabular remnant for high-dose rate brachytherapy was performed instead. The patient died 11 months after surgery without signs or symptoms of bony damage and retained the ability to walk for the remainder of her life.

* Corresponding author.

Department of Oncology, Clínica Universitaria de Navarre, University of Navarre, Avda Pío XII s/n Pamplona, Navarre, Spain.

Tel.: 34-948-255400; fax: 34-948-255500.

E-mail address: rmartinezm@unav.es (R. Martínez-Monge). 


\section{INTRODUCTION}

The patient's medical history dates back to 1996 when a localized Ewing's sarcoma of the left hemipelvis was diagnosed. She underwent a left pubectomy and then began adjuvant chemotherapy with alternating sequence vincristine-doxorubicin-ifosfamideactinomycin D (VAIA) and EVAIA (additional etoposide) that was finally supplemented with external beam radiation therapy to 55 Gy and high-dose chemotherapy with stem cell support.

In December 2000, she presented a local relapse in the left acetabulum with soft tissue extension. The biopsy confirmed the diagnosis of recurrent Ewing's sarcoma. She was placed on chemotherapy with topotecan-cyclophosphamide alternating with vincristineifosfamide-etoposide and carboplatin-etoposide, achieving a partial response. She was then referred to our institution for evaluation for salvage surgery.

On first consultation, her history and physical were unremarkable. The pelvic computed tomographic (CT) scan revealed a lesion in the left puboischiatic rami with involvement of the adjacent acetabulum. There was no soft tissue extension. The metastatic workup was negative. The bone scan revealed a diffuse area of uptake in the left hip with no other signs of metastatic disease.

\section{BRACHYTHERAPY TECHNIQUE}

In July 2001 the patient had a resection of the puboischiatic rami performed. The acetabulum was preserved, although the pretreatment magnetic resonance imaging (MRI) scan was highly suspicious for tumor involvement (Fig. 1). Five afterloading, number 6 French catheters were implanted through drill holes in the acetabular remnant (Fig. 2). The final pathology report confirmed the presence of residual Ewing's sarcoma involving the bony (intramedullary) resection margin.

On postoperative day 3, a pelvic CT scan for dosimetry was performed. The highdensity silicon inserts of the afterloading catheters were removed before this scan to allow visualization of the catheters in the bone tissue (Fig. 3).

On postoperative day 4, the brachytherapy treatment was started. Eight fractions of 4.5 Gy each prescribed at the minimum tumor dose (MTD), as described in ICRU Report 58 (1), were delivered twice daily until a total dose of 36.0 Gy was reached in 4 days, which is equivalent to an MTD of 44.5 Gy delivered in 2 Gy daily fractions if calculated for an $\alpha / \beta$ ratio of 10 with the linear quadratic formulation (2) without time correction (i.e., $\mathrm{BED}=\mathrm{nd}[1+\mathrm{d} /(\alpha / \beta])$. The treatment was completed on postoperative day 10 , and the catheters were removed uneventfully.

\section{PATIENT OUTCOME}

The patient continued on chemotherapy, but unfortunately developed lung metastases and multiple soft tissue masses in the left inguinal region 6 months after surgery. These extended upwards to the external iliac vessels and the abdominal wall. She underwent resection and perioperative high-dose rate (HDR) brachytherapy of the recurrent soft 
tissue masses in the left inguinal region. She died 11 months after surgery without signs or symptoms of bone damage in the implanted area on MRI examination.

\section{DISCUSSION}

Placement of radioactive implants in the proximity of bony structures is often discouraged due to the well-known risk of osteoradionecrosis. The American Brachytherapy Society has recently released specific recommendations in this regard (3). However, there are some instances in which implantation of lesions near or within bone tissue may be necessary for palliation or to avoid devastating surgical procedures, especially when life expectancy is short, as in recurrent Ewing's sarcoma (4).

In this report we describe the absence of clinical or radiologic bone damage after HDR brachytherapy during an observation period of 11 months, in spite of adverse factors such as delivery of prior external beam radiation over a weight-bearing bone. The patient retained the ability to walk normally for the remainder of her life. Although this strategy is not recommended for definitive primary treatment of bone sarcomas, the notion that brachytherapy should be avoided for bony lesions could be reconsidered in some specific cases.

\section{REFERENCES}

[1]. International Commission on Radiation Units, and Measurements. Dose and volume specification for reporting interstitial therapy. ICRU report 58. Bethesda, MD: International Commission on Radiation Units and Measurements; 1997.

[2]. Douglas BG, Fowler JF. The effect of multiple small doses of $\mathrm{x}$ rays on skin reactions in the mouse and a basic interpretation. Radiat Res 1976;66:401-426.

[3]. Nag S, Cano ER, Demanes DJ, et al. The American Brachytherapy Society recommendations for high-dose-rate brachytherapy for head-and-neck carcinoma. Int JRadiat Oncol Biol Phys 2001;50:1190-1198.

[4]. Rodriguez-Galindo C, Billups CA, Kun LE, et al. Survival after recurrence of Ewing tumors: The St Jude Children's Research Hospital experience, 19791999. Cancer 2002;94:561-569. 

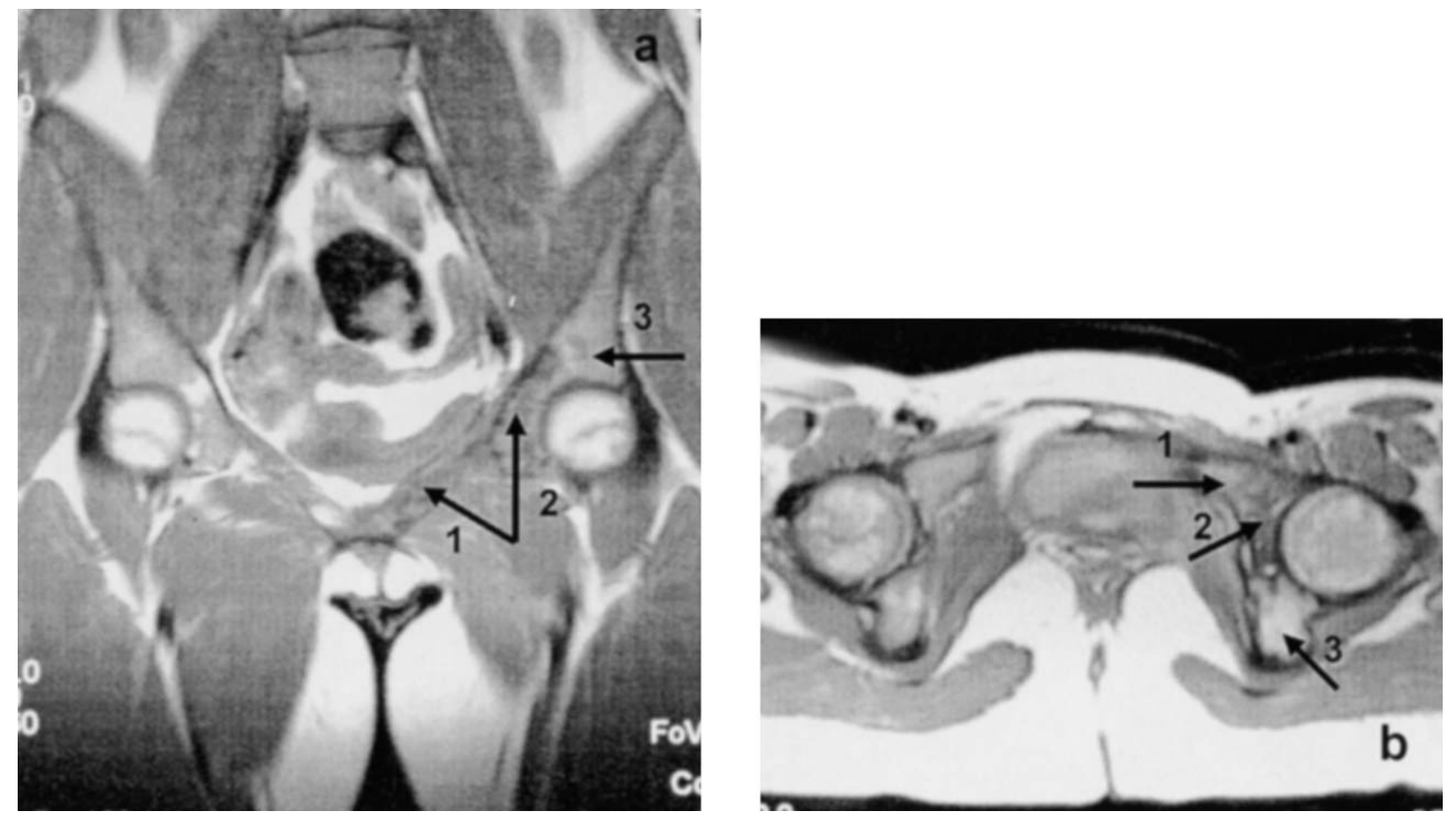

Figure 1. Coronal (a) and transverse (b) magnetic resonance imaging (MRI) scan revealing altered signal (dark) in the puboischiatic rami (arrow 1) and lower acetabulum (arrow 2). However, the upper part of the acetabulum exhibits normal MRI signal (bright; arrow 3).
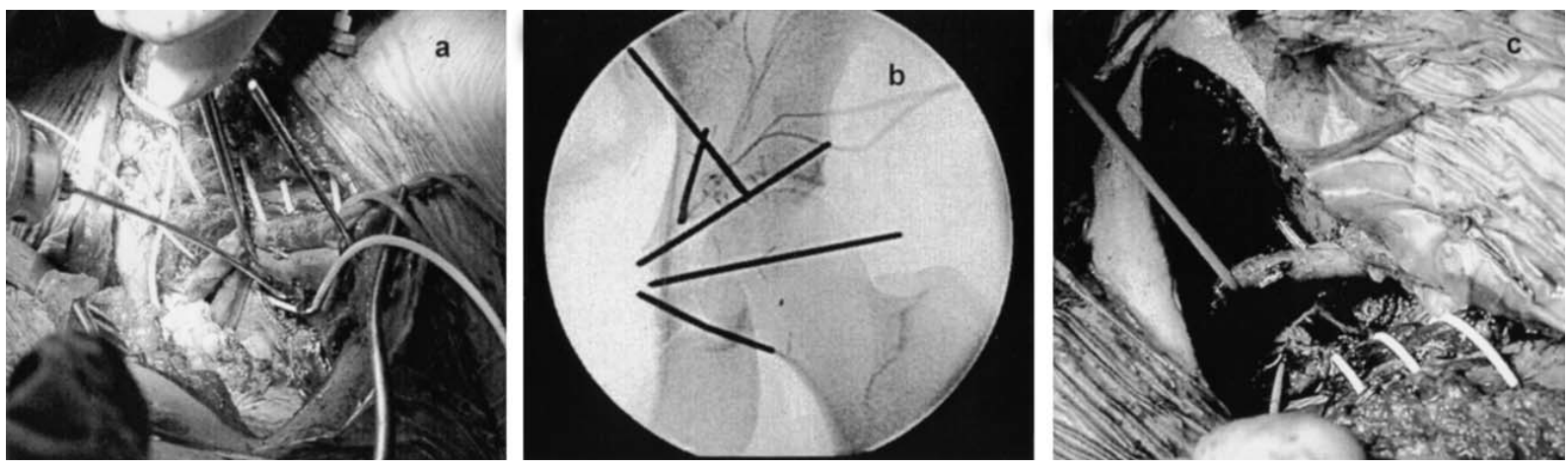

Figure 2. (a) Intraoperative view illustrating hole drilling. (b) Intraoperative X-ray film revealing drill holes in place. (c) Intraoperative view demonstrating afterloading catheters and bony resection margin (arrow). 

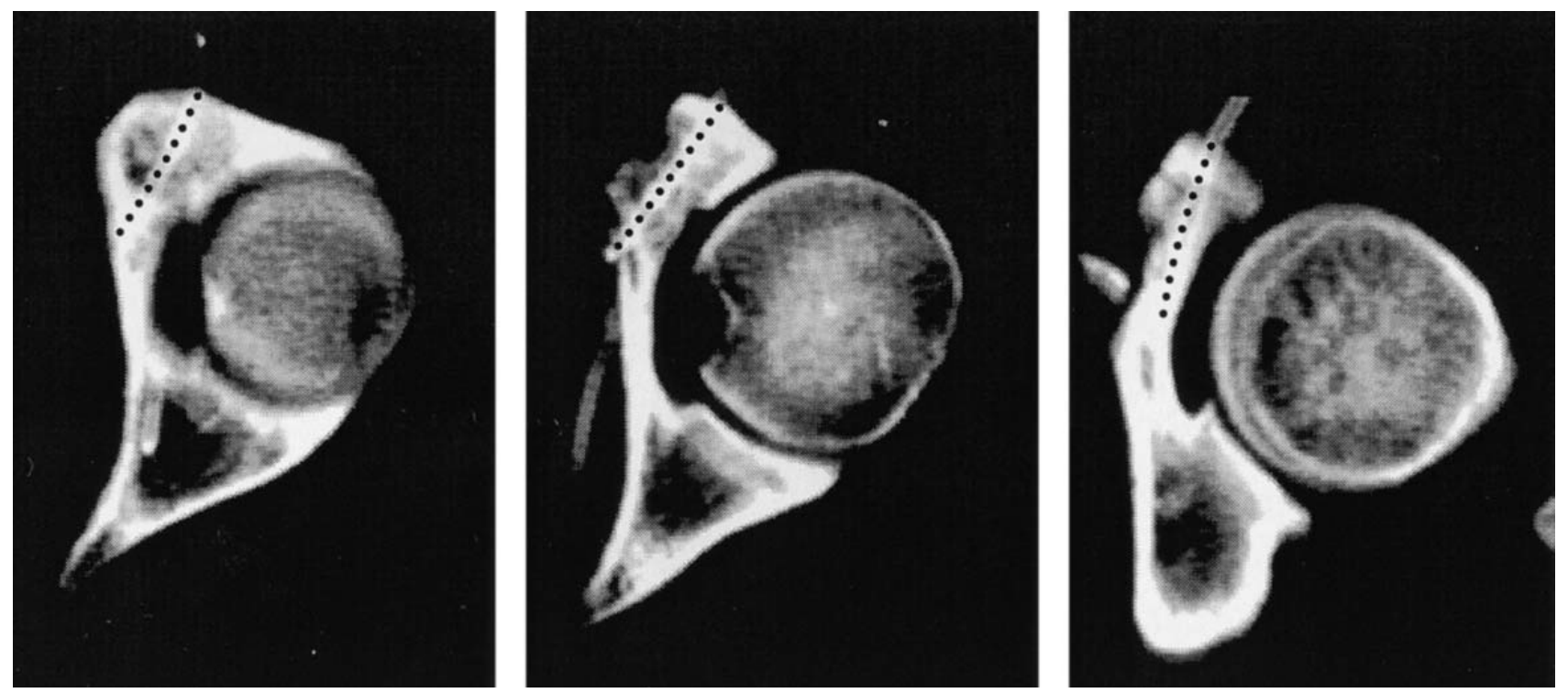

Figure 3. Bone computed tomography scan of the left acetabulum for dosimetry. Dotted lines highlight the intraosseous passage of the afterloading catheters at three different levels. 